\title{
Erosión del esmalte dental en dientes expuestos a bebidas de origen industrial. Estudio piloto in vitro.
}

\section{Erosion of dental enamel in teeth exposed to industrial drinks. In vitro pilot study.}

\author{
Margarita Trujillo-Hernández ${ }^{1}$, Annet Alondra Acosta-Acosta², María Paola Burgos Anaya ${ }^{3}$, \\ Vivi Hoyos-Hoyos ${ }^{4}$, Jennifer Orozco-Páez ${ }^{5^{*}}$
}

\begin{abstract}
1. Facultad de odontología, Universidad
Veracruzana, Veracruz, México.

2. Licenciatura en Cirujano Dentista, Instituto de Ciencias Biomédicas, Universidad Autónoma de Ciudad Juárez, Ciudad Juárez, México.

3. Semillero de Investigación SEIFO, programa de odontología, Facultad de Ciencias de la Salud, Corporación Universitaria Rafael Núñez, Cartagena, Colombia.

4. Joven Investigador - Colciencias, Programa de Odontología, Facultad de Ciencias de la Salud, Corporación Universitaria Rafael Núñez, Cartagena, Colombia.

5. Grupo de Investigación GISACC, Programa de Odontología, Facultad de Ciencias de la Salud, Corporación Universitaria Rafael Núñez, Cartagena, Colombia.

* Correspondencia Autor: Jennifer Orozco Páez | Dirección: Centro Calle de la Soledad N ${ }^{\circ} 5-70$, Edificio Rafael Núñez, 3er Piso, Oficina del Programa de Odontología. | Teléfono: +57 5 6517088. | E-mail: jennifer.orozco@curnvirtual.edu.co.

Trabajo recibido el 17/08/2020

Trabajo revisado 22/12/2020

Aprobado para su publicación el 30/12/2020
\end{abstract}

Margarita Trujillo-Hernández: https://orcid.org/00000002-2463-7909

Annet Alondra Acosta-Acosta: https://orcid. org/0000-0002-6690-4374

Vivi Hoyos-Hoyos: https://orcid.org/0000-00023085-7772

Jennifer Orozco-Páez: https://orcid.org/0000-00034834-071X

\section{RESUMEN}

Objetivo: determinar el efecto erosivo sobre el esmalte dental de dientes humanos extraídos, después de la exposición a bebidas industriales distribuidas comercialmente en Cartagena, Colombia. Materiales y métodos: estudio experimental in vitro en 26 dientes humanos extraídos con esmalte dental sano, distribuidos aleatoriamente en doce grupos experimentales que fueron expuestos a bebidas industriales comercializadas en la ciudad de Cartagena. Las bebidas fueron expuestas durante 7 días, dos veces al día. Para determinar el efecto erosivo, se valoró pH de la bebida, pérdida de peso de los dientes expuestos y aspecto clínico del esmalte dental posterior a la exposición. Resultados: todas las bebidas evaluadas presentaron $\mathrm{pH}<4.5$, siendo la Coca Cola ${ }^{\circledR}$ y jugo Hit $\circledast$ naranja las que exhibieron el pH más bajo. Todas las bebidas causaron desmineralización del esmalte, sin embargo, las que causaron mayor pérdida de peso fueron Speed Max $₫$, Vive $100 \AA$ y Coca-Cola $₫$. Conclusiones: Speed Max $₫$ y Coca-Cola $₫$ fueron las bebidas que generaron mayor efecto erosivo sobre la estructura dental, por lo cual es necesario informar a la población los riesgos para la salud oral que se derivan del consumo desmedido de estas bebidas.

PALABRAS CLAVE

Esmalte dental; Erosión dental; Bebidas; Ingestión de líquidos salud pública (DeCS).

Int. J. Inter. Dent Vol. 14(3); 237-241, 2021.

\begin{abstract}
Objective: to determine the erosive effect on dental enamel of extracted human teeth, after exposure to industrial drinks distributed commercially in Cartagena, Colombia. Materials and methods: experimental in vitro study in 26 human teeth extracted with healthy dental enamel, randomly distributed in twelve experimental groups that were exposed to industrial beverages commercialized in the city of Cartagena. The teeth were exposed to the drinks for 7 days, twice a day. To determine the erosive effect, $\mathrm{pH}$ of the drink, weight loss of the exposed teeth and clinical appearance of the dental enamel after exposure were evaluated. Results: all the evaluated drinks presented $\mathrm{pH}<4.5$, being Coca $\mathrm{Cola}^{\circledR}$ and $\mathrm{Hit}^{\circledR}$ Orange Juice the ones that exhibited the lowest $\mathrm{pH}$. All the drinks caused enamel demineralization, however, the ones that caused the most weight loss were Speed Max ${ }^{\circledR}$, Vive $100^{\circledR}$ and Coca Cola ${ }^{\circledR}$. Conclusions: Speed $\mathrm{Max}^{\circledR}$ and Coca Cola ${ }^{\circledR}$ were the drinks that generated the greatest erosive effect on the dental structure. Therefore, it is necessary to inform the population about the risks to oral health derived from the excessive consumption of these drinks.
\end{abstract}

KEY WORDS

Dental enamel; Dental erosion; Drinks; Public health fluid intake (DeCS).

Int. J. Inter. Dent Vol. 14(3); 237-241, 2021.

\section{INTRODUCCIÓN}

El consumo de bebidas procesadas ha aumentado exponencialmente en las últimas décadas por su fácil y rápido acceso. Colombia, se ubica entre los mayores consumidores mundiales de bebidas azucaradas; específicamente de bebidas carbonatadas. Datos de la última encuesta de situación nutricional en Colombia (ENSIN-2015) muestran que estos productos ocupan el octavo lugar con un volumen promedio de consumo diario de $373,5 \mathrm{~g}^{(1)}$.

Una amplia gama de estos productos se distribuye en diferentes presentaciones con la intención de dirigirse a diversos sectores de la población y satisfacer necesidades variadas, las más comunes son las bebidas energizantes, refrescantes, carbonatadas, hipocalóricas, multivitamínicas, entre otras ${ }^{(2)}$. Ya se ha demostrado que el consumo crónico de la mayoría de bebidas procesadas genera efectos nocivos en el organismo. Específicamente a nivel oral, el consumo no controlado de estas bebidas se ha asociado con la alteración del $\mathrm{pH}$ salival y por lo tanto con procesos de desmineralización del esmalte y erosión dental $(3,4)$

La erosión dental se define como la pérdida patológica, crónica, localizada, indolora, de los tejidos dentales por acción química 
de ácidos y/o quelantes, no asociados a los producidos por la flora bacteriana que origina la caries dental o por factores mecánicos o traumáticos ${ }^{(5)}$. Es una alteración cuya tasa de incidencia ha venido aumentando con los años, esto se debe a que no solamente se relaciona con trastornos gastroesofágicos, bulimia o anorexia, sino también con el consumo desmedido de determinados alimentos o bebidas, sobre todo los de origen industrial $(6,7)$.

Shroff $\mathrm{P}$ y cols $^{(8)}$ concluyeron después de evaluar el potencial erosivo de veinte bebidas en dientes humanos, que existió una diferencia significativa $(p<0.05)$ en el porcentaje de pérdida de peso después de 6 y 24 horas de inmersión.

Teniendo en cuenta estos antecedentes, es necesario identificar qué tipo de bebidas procesadas poseen mayor potencial erosivo con la finalidad de informar y educar a la población respecto a los riesgos que representa su consumo desmedido.

El objetivo de este estudio fue determinar el efecto erosivo sobre el esmalte dental de dientes extraídos, después de la exposición a bebidas industriales distribuidas comercialmente en Cartagena, Colombia.

\section{MATERIALES Y MÉTODOS}

Se realizó un estudio experimental in vitro cuya muestra estuvo conformada 26 dientes humanos extraídos, obtenidos mediante donación voluntaria y firma de consentimiento informado por parte del paciente donante. Se incluyeron dientes íntegros con esmalte dental sano y se excluyeron dientes con restauraciones dentales extensas, dientes con caries dental, anomalías del desarrollo o con manchas externas del esmalte.

Los dientes se distribuyeron aleatoriamente en doce grupos experimentales que fueron expuestos a bebidas procesadas comercializadas en la ciudad de Cartagena - Colombia. Los grupos quedaron conformados de la siguiente manera: (Grupo 1: Coca-Cola ${ }^{\circledR}$ (tradicional); Grupo 2: manzana Postobón ${ }^{\circledR}$; Grupo 3: Postobón ${ }^{\circledR}$ naranja; Grupo 4: Postobón ${ }^{\circledR}$ uva; Grupo 5: Kola Román ${ }^{\circledR}$; Grupo 6: Pony malta ${ }^{\circledR}$; Grupo 7: jugo Hit ${ }^{\circledR}$ sabor mora; Grupo 8: jugo Hit ${ }^{\circledR}$ naranja; Grupo 9: Speed Max ${ }^{\circledR}$; Grupo 10: Vive 100 ${ }^{\circledR}$; Grupo 11: Agua con gas Brisa ${ }^{\circledR}$ lima-limón) y un grupo control (Grupo 12) expuesto a saliva artificial (Salivar ${ }^{\circledR}$, Farpag). Cada grupo de estudio estuvo conformado por 2 dientes, al grupo control se le asignaron 4 dientes.

Para determinar el efecto erosivo, se valoró el pH de la bebida, la pérdida de peso de los dientes expuestos y el aspecto clínico del esmalte dental posterior a la exposición.

Preparación de los dientes: previo al protocolo de exposición, los dientes fueron sometidos a un proceso de limpieza para eliminar restos orgánicos e inorgánicos adheridos a su superficie. Se utilizaron curetas de Gracey ${ }^{\circledR}$ y agua destilada para el lavado. Cada diente fue pesado en una balanza analítica de semiprecisión (Adam AFP-110L).

Posteriormente, se evaluó el brillo y textura del esmalte, se le realizó un registro fotográfico para dejar evidencia del estado inicial de cada diente. El estudio fotográfico realizado en cada grupo de dientes, se realizó bajo las siguientes especificaciones: cámara Reflex os Rebel TG, objetivo 18-55mm (distancia focal); configuración: tiempo de exposición: 1/100 segundos, apertura de diafragma: $\mathrm{f} 22$, sensibilidad (iso): 100; modo de enfoque: manual, modo de disparo: manual, -calidad de imagen: $L$ (5184 X 3456 pixeles), caja de luz LED / fondo negro-gris (neutro), distancia objetivo - muestra: $30 \mathrm{cms}$.

De cada bebida procesada se tomaron muestras (se midió el pH de 6 lotes diferentes de cada bebida, las lecturas se realizaron por duplicado) y se midió su pH mediante un $\mathrm{pH}$ metro digital portátil (Ref. 315i marca WTW), de esta manera se obtuvo un duplicado de lectura de $\mathrm{pH}$ que fue promediada para obtener el $\mathrm{pH}$ final.

Exposición de dientes a bebidas: el protocolo de exposición fue adaptado del estudio de Liñan C y cols $^{(9)}$, cada grupo de dientes se expuso a las bebidas correspondientes dos veces al día durante 7 días para obtener un total de 14 exposiciones. La exposición consistió en la inmersión de los dientes en la bebida durante 1 minuto, seguido de una inmersión en saliva artificial durante 3 minutos; estos ciclos se repitieron 5 veces para obtener un total de 20 minutos de exposición. Las bebidas fueron renovadas diariamente y se les realizó medición de $\mathrm{pH}$ a cada bebida nueva con un $\mathrm{pH}$ metro digital. Los dientes se almacenaron en recipientes limpios humedecidos en saliva artificial, y se determinó su peso en la balanza analítica todos los días después de ser expuestos a las bebidas.

Al finalizar el proceso de exposición, se evaluaron las características macroscópicas del esmalte y se determinó el peso final de cada diente.

Los datos fueron tabulados en una tabla de Excel 2019, luego se exportaron al software estadístico SPSS $^{\circledR}$, se analizaron inicialmente mediante estadística descriptiva utilizando promedios y porcentajes, para la comparación de pesos se aplicaron pruebas de normalidad, los datos fueron paramétricos por lo tanto, para comparar el peso de los dientes antes y después de la exposición, se aplicó una prueba T-student para muestras relacionadas, asumiendo diferencia estadísticamente significativa cuando el valor de $p<0,05$. Para realizar la comparación de $\mathrm{pH}$ y porcentaje de pérdida de peso entre los diferentes tipos de bebidas, se aplicó un test ANOVA con Post hoc de Bonferroni, se asumió diferencia estadísticamente significativa cuando el valor de $p<0,05$.

Consideraciones éticas: el protocolo de estudio fue aprobado por el comité de ética de la Corporación Universitaria Rafael Núñez. Así mismo, a los donantes de los dientes se les explicó el objeto del estudio por medio del consentimiento informado el cual tuvo en consideración las normas éticas de la Declaración de Helsinki y la resolución 8430 de 1993 del Ministerio de Salud de la República de Colombia.

\section{RESULTADOS}

Todas las bebidas evaluadas presentaron un $\mathrm{pH}<4,5$ siendo la

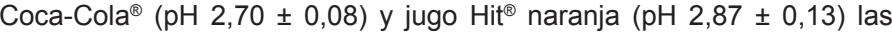
que exhibieron el $\mathrm{pH}$ más bajo. Contrario a esto la bebida con menos ácido fue Pony malta ${ }^{\circledR}$ con un $\mathrm{pH}$ de $4,07 \pm 0,04$ (Tabla 1). Al cruzar y comparar los valores de $\mathrm{pH}$ de las distintas bebidas (estadístico ANOVA con Post hoc de Bonferroni), se encontró diferencia estadísticamente significativa $(p<0,05)$ en la mayoría de los cruces. Se observó diferencia estadísticamente significativa entre el $\mathrm{pH}$ de la saliva artificial y todas las bebidas evaluadas $(p=0,000)$; asimismo, la Pony Malta ${ }^{\circledR}$ presentó diferencia estadística significativa con el resto de bebidas; la Coca-cola ${ }^{\circledR}$ por su parte, siendo la bebida con el pH más bajo, presentó diferencia significativa $(p<0,05)$ con todas las bebidas a excepción de Naranja Postobón $^{\circledR}$, Speed Max ${ }^{\circledR}$ y Hit Naranja ${ }^{\circledR}$ que también tuvieron un $\mathrm{pH}$ inferior a 3.0 .

Tabla 1: $\mathrm{pH}$ de bebidas evaluadas

\begin{tabular}{|c|c|c|}
\hline Tipo de bebida & Bebida & $\mathrm{pH}($ media $\pm \mathrm{DS})$ \\
\hline Agua saborizada & $\begin{array}{c}\text { Agua con gas Brisa }{ }^{\circledR} \\
\text { Lima - Limón }\end{array}$ & $2,92 \pm 0,04$ \\
\hline \multirow{6}{*}{$\begin{array}{l}\text { Bebidas } \\
\text { carbonatadas }\end{array}$} & Coca - Cola $\AA$ & $2,70 \pm 0,08$ \\
\hline & Pony Malta® & $4,07 \pm 0,04$ \\
\hline & Uva Postobón® & $2,98 \pm 0,08$ \\
\hline & Kola Román® & $3,25 \pm 0,05$ \\
\hline & Naranja Postobón® & $2,89 \pm 0,06$ \\
\hline & Manzana Postobón® & $3,27 \pm 0,04$ \\
\hline \multirow{2}{*}{ Bebidas energizantes } & Speed Max $®$ & $2,98 \pm 0,05$ \\
\hline & Vive $100 \AA$ & $3,16 \pm 0,04$ \\
\hline \multirow{2}{*}{ Jugos procesados } & Jugo Hit $®$ Mora & $3,20 \pm 0,05$ \\
\hline & Jugo Hit@ Naranja & $2,87 \pm 0,13$ \\
\hline Control & $\begin{array}{l}\text { Saliva artificial } \\
\text { Salivar® }\end{array}$ & $5,80 \pm 0,05$ \\
\hline
\end{tabular}

Después de la exposición a las bebidas, se observó pérdida de brillo y signos de desmineralización en el esmalte y cemento de todos los grupos a excepción del grupo de Pony malta ${ }^{\circledR}$ y el grupo control. La bebida más erosiva fue la bebida energizante Speed Max $^{\circledR}$, que a la sexta exposición causó desmineralización, seguido de la Coca-Cola ${ }^{\circledR}$, cuyo efecto erosivo fue notorio desde la séptima exposición, causando adicionalmente pigmentación. Por otro lado, las bebidas de la marca Postobón ${ }^{\circledR}$ y jugo $\mathrm{Hit}^{\circledR}$ además de generar un efecto erosivo evidente, pigmentaron la superficie dental, mayormente notoria en el cemento. Por su parte, la Pony malta ${ }^{\circledR}$ no generó desmineralización evidente en el esmalte, los dientes expuestos a esta bebida conservaron un esmalte brillante, sin signos de alteración. Asimismo, los dientes expuestos a saliva artificial no experimentaron cambios evidentes (Figura 1).

Con relación a las variaciones de peso de los dientes, todos los grupos experimentales presentaron pérdida de peso, con un rango de $0,04 \mathrm{mg}$ para Pony malta ${ }^{\circledR}$ con el valor mínimo de pérdida, hasta 7.78 mg para Speed Max ${ }^{\circledR}$ con el valor máximo. Dentro de los dientes que experimentaron las más altas pérdidas de peso fueron el premolar expuesto a Vive $100^{\circledR}$, el canino expuesto a Manzana Postobón ${ }^{\circledR}$ y el central superior expuesto a Coca-Cola ${ }^{\circledR}$ (Tabla 2). 


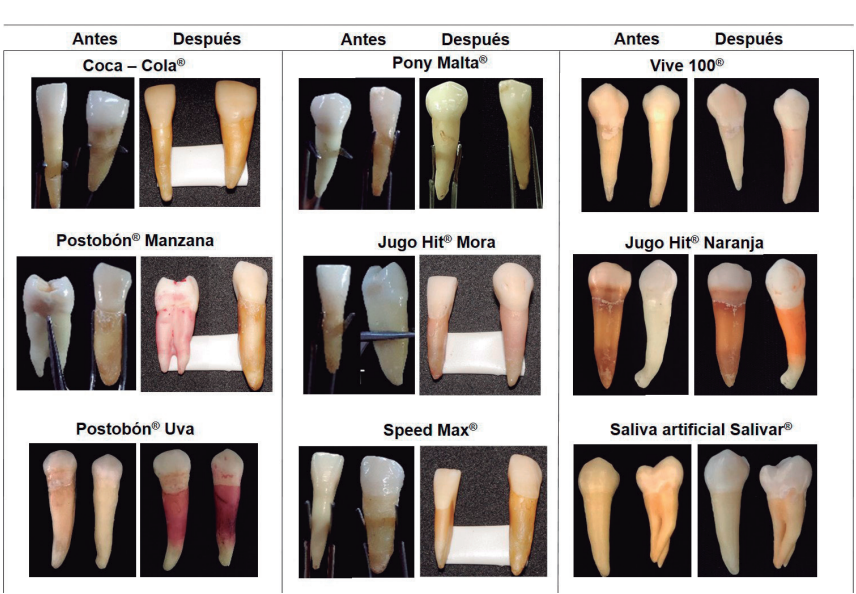

$\overline{\text { Figura 1. Características macroscópicas del esmalte y cemento dental, }}$ antes y después de la exposición a bebidas.

Para estimar la importancia de la pérdida de peso, se realizó una comparación entre los valores obtenidos antes y después de la exposición, observándose diferencia estadísticamente significativa para todos los grupos de bebidas (Tabla 3).

Al comparar las medias de pérdida de peso para cada bebida se observó diferencia estadísticamente significativa, siendo Vive $100^{\circledR}$, jugo $\mathrm{Hit}^{\circledR}$ naranja y Coca-Cola ${ }^{\circledR}$ las bebidas cuyos dientes presentaron un promedio mayor de pérdida de peso después de la exposición.
Tabla 3: Comparación de medias de peso antes y después de la exposición a las bebidas.

\begin{tabular}{l|c|c}
\hline & MEDIA $^{*} \pm \mathrm{DS}$ & \\
\hline ANTES & $1173,6 \pm 351,8$ & \multirow{2}{*}{$\mathrm{p}=0,000^{\star *}$} \\
\hline DESPUÉS & $1147,9 \pm 404,1$ & \\
\hline
\end{tabular}

${ }^{*}$ Promedio de pesos de todos los dientes expuestos a los diferentes grupos de bebidas antes y después de la exposición

${ }^{*}$ Prueba T para muestras relacionadas

\section{DISCUSIÓN}

Los reportes de literatura indican que un gran porcentaje de las lesiones erosivas del esmalte se relacionan directamente con el consumo de alimentos, incluyéndose en este grupo las bebidas industrializadas, las cuales, han sido diversificadas considerablemente en los últimos años ${ }^{(10,11)}$. En este proceso de diversificación se han relacionado como factores de riesgo para la salud sistémica y oral. En el caso de la salud oral, el consumo de estas bebidas representa un factor de riesgo para la desmineralización y erosión del esmalte dental $(12,13,14)$. Es por ello que el propósito de este estudio se centró en la evaluación del efecto erosivo de bebidas distribuidas localmente en Cartagena de Indias. Uno de los resultados más relevantes de este estudio, pone de manifiesto que variables como el $\mathrm{pH}$ de la bebida, la frecuencia de consumo y extensión del hábito de consumo en el tiempo, son factores que influyen de manera decisiva en el proceso de desmineralización del esmalte dental. Distintos autores señalan factores adicionales que también juegan un papel central.

Tabla 2: Relación de valores de pérdida de peso en dientes expuestos.

\begin{tabular}{|c|c|c|c|c|c|c|}
\hline \multirow{2}{*}{ Tipo de bebida } & \multirow{2}{*}{ Bebida } & \multirow{2}{*}{ Diente } & \multicolumn{2}{|c|}{ Peso promedio (mg). } & \multirow{2}{*}{$\begin{array}{l}\text { Pérdida de peso } \\
\text { absoluto (mg) }\end{array}$} & \multirow{2}{*}{$\begin{array}{l}\text { Promedio \% } \\
\text { Pérdida de peso }\end{array}$} \\
\hline & & & Antes & Después & & \\
\hline \multirow{2}{*}{ Agua saborizada } & \multirow{2}{*}{$\begin{array}{l}\text { Agua con gas } \\
\text { lima-limón }\end{array}$} & Premolar & 1342,2 & 1339,2 & 3 & \multirow{2}{*}{$0,15^{*}$} \\
\hline & & Molar & 2043,1 & 2041,7 & 1,4 & \\
\hline \multirow{12}{*}{$\begin{array}{l}\text { Bebidas } \\
\text { carbonatadas }\end{array}$} & \multirow{2}{*}{ Coca Cola ${ }^{\circledR}$} & ${ }^{*} \mathrm{C}$. Superior & 962,24 & 956,93 & 5,31 & \multirow{2}{*}{$0,57 \pi$} \\
\hline & & C. Inferior & 713,69 & 709,55 & 4,14 & \\
\hline & \multirow{2}{*}{ Pony Malta ${ }^{\circledR}$} & Premolar & 1077,6 & 1076,25 & 1,34 & \multirow{2}{*}{$0,07 ¥$} \\
\hline & & ${ }^{*}$ L. Inferior & 706,38 & 706,34 & 0,04 & \\
\hline & \multirow{2}{*}{ Postobón® Uva } & Premolar & 1002,8 & 999,7 & 3,1 & \multirow{2}{*}{0,28} \\
\hline & & Canino & 1178,55 & 1175,55 & 3 & \\
\hline & \multirow{2}{*}{ Kola Román ${ }^{\circledR}$} & C. inferior & 750,2 & 748,75 & 1,45 & \multirow{2}{*}{$0,14 £$} \\
\hline & & Premolar & 1142,7 & 1141,65 & 1,05 & \\
\hline & \multirow{2}{*}{$\begin{array}{l}\text { Postobón }{ }^{\circledR} \\
\text { Naranja }\end{array}$} & Premolar & 1198,3 & 1193,7 & 4,55 & \multirow{2}{*}{0,37} \\
\hline & & Premolar & 1250,7 & 1246,2 & 4,5 & \\
\hline & \multirow{2}{*}{$\begin{array}{l}\text { Manzana } \\
\text { Postobón® }\end{array}$} & Premolar & 1267,2 & 1262,3 & 4,82 & \multirow{2}{*}{0,39} \\
\hline & & Canino & 1685,3 & 1678,5 & 6,72 & \\
\hline \multirow{4}{*}{$\begin{array}{l}\text { Bebidas } \\
\text { energizantes }\end{array}$} & \multirow{2}{*}{ Speed Max® } & Canino & 1213,1 & 1205,35 & 7,71 & \multirow{2}{*}{$0,88 \#$} \\
\hline & & C. Inferior & 504 & 498,29 & 5,71 & \\
\hline & \multirow{2}{*}{ Vive $100 \AA$} & Premolar & 1036,2 & 1030,5 & 5,7 & \multirow{2}{*}{0,51} \\
\hline & & Premolar & 1315,35 & 1309,05 & 6,3 & \\
\hline \multirow{4}{*}{$\begin{array}{l}\text { Jugos } \\
\text { procesados }\end{array}$} & \multirow{2}{*}{ Jugo Hit® Mora } & Premolar & 1312,49 & 1311,05 & 1,44 & \multirow{2}{*}{0,37} \\
\hline & & C. Inferior & 545,13 & 541,66 & 3,47 & \\
\hline & \multirow{2}{*}{$\begin{array}{l}\text { Jugo Hit@ } \\
\text { Naranja }\end{array}$} & Premolar & 1274,7 & 1268,5 & 6,2 & \multirow{2}{*}{0,4} \\
\hline & & Premolar & 1684,05 & 1678,9 & 5,15 & \\
\hline Control & $\begin{array}{l}\text { Saliva artificial } \\
\text { Salivar }{ }^{\circledR}\end{array}$ & Molar & 1218,3 & 1219,8 & $-1,5$ & $-0,10+$ \\
\hline
\end{tabular}

${ }^{*} C$ (Incisivo Central); * (Incisivo Lateral). Estadístico ANOVA con Post hoc Bonferroni, los valores entre los que se presentó diferencia estadísticamente significativa con un p<0,05 fueron (* vs $\#)$, ( $\pi$ vs + ), (¥vs \#), (£vs \#), (\#vs +), (\#vs*). 
Un ejemplo concreto es el estudio prospectivo publicado por Hasselkvist A y cols ${ }^{(15)}$, cuyo objetivo fue evaluar la progresión de la erosión dental en niños de 13 a 14 años durante 4 años y su asociación con el estilo de vida y la salud bucal. Evaluaron 2566 superficies de dientes en total, de las cuales, el 35\% experimentó progresión de la lesión erosiva. Dos de las variables que predijeron una mayor progresión de la lesión erosiva fue "El hábito de retención de bebidas ácidas en boca antes de tragar" y el "consumo frecuente de bebidas entre comidas".

Así mismo, Marqués Martínez $\mathrm{L}^{(16)}$ determinaron la relación entre la prevalencia de la erosión dental en 400 niños de 6 a 14 años y todos los factores etiológicos que conducen al desarrollo de las lesiones mediante el índice BEWE (Basic Erosion Wear Examination) y el riesgo de erosión para cada paciente. Encontraron una prevalencia del $22,3 \%$ y se observó una correlación positiva entre la presencia de erosión dental y la ingesta frecuente de jugos de frutas, bebidas carbonatadas e isotónicas ( $p$ $<0,05)$, presentando una correlación más alta si el líquido se mantuvo en la boca antes de tragar.

Teniendo en cuento los reportes anteriormente mencionados, el hábito de retener la bebida en boca antes de ingerirla y el momento de consumo de la bebida (con las comidas o entre comidas) son variables que emergen en el proceso y deben ser objeto de estudio en aras de dilucidar todos los factores que otorgan el potencial erosivo a las bebidas de origen industrial.

Los reportes de la literatura indican que el potencial erosivo de las bebidas depende de múltiples factores, dentro de ellos, el tipo de ácido que contiene, su concentración, temperatura, $\mathrm{pH}$, tiempo de permanencia de la bebida en la boca y la capacidad buffer salival del huésped son los más reportados ${ }^{(17,18)}$. En nuestro estudio, todas las bebidas evaluadas son de alta comercialización y de consumo frecuente en la población universitaria y presentaron $\mathrm{pH}<5,0$, siendo éste un $\mathrm{pH}$ muy inferior al $\mathrm{pH}$ normal de la saliva $(\mathrm{pH}: 6.2-7.4)$ e inferior al $\mathrm{pH}$ considerado crítico para la disolución del esmalte ( $\mathrm{pH}: 5.5)$, por lo tanto, la presencia de estas bebidas ácidas de manera prolongada en la cavidad oral, tiene la capacidad de generar un aumento en la concentración de iones $\mathrm{H}+\mathrm{y}$ por consiguiente el descenso del $\mathrm{pH}$ salival, lo que puede desencadenar procesos de desmineralización y pérdida de estructura dental si el consumo es muy frecuente ${ }^{(19,20)}$.

El seguimiento realizado a los dientes, demostró que el efecto erosivo y la alteración del peso fue diferente entre los grupos a pesar de que todas las bebidas presentaron un $\mathrm{pH}$ ácido. Se observó que los dientes expuestos a Coca-Cola ${ }^{\circledR}$ y bebidas energizantes (Vive $100^{\circledR}$ y Speed Max ${ }^{\circledR}$ ) experimentaron de manera más temprana pérdida de brillo del esmalte y fueron los dientes con mayor pérdida de peso al final de la exposición, lo cual presenta concordancia con lo expuesto por de Medeiros, R. A y cols $^{(21)}$ quienes afirman que el principal potencial erosivo en las bebidas es el $\mathrm{pH}$, mientras que el tipo de ácido, la acidez titulable, el potencia quelante, la concentración de calcio, fosfato y flúor, son factores que inciden en el proceso erosivo.

Contrastando nuestros resultados, Barac $R$, et $a^{(22)}$ evaluaron el potencial erosivo de algunas bebidas teniendo en cuenta el $\mathrm{pH}$ y la acidez titulable, así como la rugosidad de la superficie del esmalte en seis grupos (Coca-Cola ${ }^{\circledR}$, jugo de naranja, Cedevita ${ }^{\circledR}$, Guaraná, Yogurt de fresa y un grupo control) en 96 dientes, los cuales fueron expuestos 3 veces al día, durante 10 días, encontrando que la Coca-Cola ${ }^{\circledR}$ tuvo un alto potencial durante los 15 minutos de exposición, mientras que el yogurt de fresa no causó erosión sin tener en cuenta el tiempo de exposición, estos resultados fueron similares a los nuestros.

Los componentes que participan en la acidificación de las bebidas industriales que aumentan su potencial erosivo son: 1. Dióxido de carbono: responsable del efecto de burbujeo del refresco, este gas se inyecta en la bebida bajo presión, a medida que se agrega más dióxido de carbono, disminuye el $\mathrm{pH}$, dando más acidez al refresco. 2. Ácidos: la mayoría de los refrescos contienen ácidos, los más comunes son el ácido cítrico y el ácido fosfórico. Estos ácidos proporcionan esa sensación refrescante y al mismo tiempo preservan la calidad y neutralizan la dulzura de la bebida ${ }^{(23)}$ de Medeiros, R. A y otros ${ }^{(24)}$ afirman que el principal potencial erosivo en las bebidas es el $\mathrm{pH}$, mientras que el tipo de ácido, la acidez titulable, el potencial quelante, la concentración de calcio, fosfato y flúor son factores que influyen en el proceso erosivo.

Estos componentes le dan a la bebida un $\mathrm{pH}$ que generalmente es menor a 3.5. Debido a que el pH salival varía de 5.5 a 7.0, la exposición prolongada a este $\mathrm{pH}$ bajo, con frecuencia puede resultar en una desmineralización rápida del esmalte.

Respecto a la variación en la masa de los dientes, los dientes expuestos presentaron disminución de peso después de la exposición a las bebidas, siendo la excepción el grupo control expuesto a saliva artificial y el grupo expuesto a Pony Malta $^{\circledR}$ que no experimentaron pérdida de peso, así como tampoco se observó clínicamente erosión del esmalte dental en los dientes expuestos a esta bebida, lo que nos permitió corroborar que la pérdida de peso se relaciona directamente con la pérdida de minerales que experimenta el diente.

Por otro lado, los análisis estadísticos demostraron que, con tan solo siete días de exposición, la pérdida de peso que experimentaron los dientes fue significativa $(0,4$ a $0,8 \%)$. De ello se deriva una importante consideración, los consumidores frecuentes se encuentran expuestos a diario y más de una vez al día al potencial erosivo de estas bebidas, es normal que en esta población la ingesta se extienda en el tiempo sobrepasando un consumo de más de siete días consecutivos, lo que aumenta la probabilidad de que se supere la capacidad amortiguadora de la saliva conllevando a la desmineralización del esmalte y progresivamente a la aparición de lesiones erosivas.

Este supuesto es respaldado por el estudio In Vitro de Gravelle BL y cols ${ }^{(25)}$, quienes expusieron durante 20 días, 42 molares sanos con el fin de determinar el potencial erosivo de diversas bebidas gaseosas de consumo común. Los dientes fueron pesados antes y después de la exposición reportando porcentajes de pérdida de peso desde $3,22 \%$ a $44,52 \%$, al comparar estos datos con nuestros resultados, se observa que el porcentaje de pérdida de peso corresponde y se relaciona con el número de días de exposición de la estructura dentaria a la bebida.

Actualmente, un gran número de universitarios y la población en general tienen como hábito el consumo frecuente de bebidas industriales y desconocen sus efectos en la salud oral, por lo tanto, la intervención desde el profesional en formación hasta el odontólogo titulado juega un rol trascendental en la educación de los pacientes y reducción de los riesgos derivados del consumo de este tipo de bebidas.

Todas las bebidas evaluadas presentaron un $\mathrm{pH}<4,5$ lo cual les confiere potencial erosivo. De igual forma, estas bebidas generaron pérdida macroscópica de brillo sobre la superficie del esmalte dental atribuible a pérdida de minerales, a excepción del grupo control y el grupo expuesto a Pony malta ${ }^{\circledR}$. Por último, los dientes de todos los grupos experimentales presentaron pérdida de peso, siendo los dientes expuestos a Coca-Cola ${ }^{\circledR}$, Vive $100^{\circledR}$, Speed Max ${ }^{\circledR}$ y jugo $\mathrm{Hit}^{\circledR}$ naranja los que experimentaron un mayor porcentaje de pérdida.

\section{RELEVANCIA CLÍNICA}

\section{Justificación científica para el estudio:}

El consumo de bebidas procesadas se ha incrementado en la población mundial, y su consumo descontrolado se ha categorizado como un factor de riesgo para la erosión dental.

Resultados principales:

Todas las bebidas evaluadas presentaron un $\mathrm{pH}<4,5$ lo cual les confiere potencial erosivo. Los dientes experimentaron pérdida de peso relacionada con la pérdida de minerales del esmalte dental.

\section{Consecuencias prácticas:}

El asesoramiento por parte del odontólogo a la población de pacientes respecto al consumo de productos industriales, debe basarse en evidencia científica, con el fin de generar un consumo consciente y reducir los riesgos derivados de su consumo.

\section{CONFLICTO DE INTERÉS}

Los autores, declaran por este medio que no existen conflictos de intereses asociados a este estudio. 


\section{Bibliografía}

1. Ministerio de Salud y Protección Social, Instituto Nacional de Salud, ICBF, Universidad Nacional de Colombia. Encuesta nacional de la situación nutricional en Colombia - ENSIN 2015 [Internet]. 2015 [citado 24/02/2020]. Disponible en: www. icbf.gov.co

2. Kanzow P, Wegehaupt FJ, Attin T, Wiegand A. Etiology and pathogenesis of dental erosion. Quintessence Int. 2016;47(4):275-8. doi: 10.3290/j.qi. a35625.

3. Panda A, Ghosh B, Pal I, Kumar V, Bhuyan L, Dash KC. Dissolution of ename on exposure to various commercial beverages available in India. J Contemp Dent Pract. 2017;18(11):1009-13. doi: 10.5005/jp-journals-10024-2166.

4. Søvik JB, Skudutyte R, Tveit AB, Sandvik L, Mulic A. Sour sweets and acidic beverage consumption are risk indicators for dental erosion. Caries Res. 2015;49(3):243-50. doi: 10.1159/000371896.

5. Jameel RA, Khan SS, Abdul Rahim ZH, Bakri MM, Siddiqui S. Analysis of dental erosion induced by different beverages and validity of equipment for identifying early dental erosion, in vitro study. J Pak Med Assoc. 2016;66(7):843-8

6. Warreth A, Abuhijleh E, Almaghribi MA, Mahwal G, Ashawish A. Tooth surface loss: A review of literature. Saudi Dent J. 2020;32(2):53-60. doi: 10.1016/j. sdentj.2019.09.004

7. Ngoc CN, Donovan TE. Education about dental erosion in U.S. and Canadian dental schools. J Dent Educ. 2018;82(12):1296-304. doi: 10.21815/JDE.018.140.

8. Shroff P, Gondivkar SM, Kumbhare SP, Sarode S, Gadbail AR, Patil S. Analyses of the erosive potential of various soft drinks and packaged fruit juices on teeth. $J$ Contemp Dent Pract. 2018;19(12):1546-51.

9. Liñan C, Meneses A, Delgado L. Evaluación in vitro del efecto erosivo de tres bebidas carbonatadas sobre la superficie del esmalte dental. Rev Estomato Herediana. 2007; 17(2):58-62.

10. Mitic AD, Gasic JZ, Barac RG, Radenkovic GS, Sunaric SM, Popovic JZ, et al. Ultrastructural changes in the cemento-enamel junction caused by acidic beverages: An in vitro study. Microsc Res Tech. 2019;83(2):91-8. doi: 10.1002/jemt.23392.

11. Bonilla EF, Méndez C, Ibatá L. Consumo de bebidas energizantes en una población de escolares de Bogotá, Colombia. Rev Salud Pública. 2018;20(5):57983. doi: $10.15446 /$ rsap.v20n5.67430

12. Rajeev G, Lewis AJ, N S. A time based objective evaluation of the erosive effects of various beverages on enamel and cementum of deciduous and permanent teeth. J Clin Exp Dent. 2020;12(1):e1-e8. doi: 10.4317/jced.55910.

13. Hasselkvist A, Johansson A, Johansson AK. A 4-year prospective longitudina study of progression of dental erosion associated to lifestyle in $13-14$ year - old Swedish adolescents. J Dent. 2016;47:55-62. doi: 10.1016/j.jdent.2016.02.002.
14. Atalay C, Ozgunaltay G. Evaluation of tooth wear and associated risk factors: $A$ matched case-control study. Niger J Clin Pract. 2018;21(12):1607-14. doi: 10.4103/ njcp.njcp 20318.

15. Alkattan R, Lippert F, Tang Q, Eckert GJ, Ando M. The influence of hardness and chemical composition on enamel demineralization and subsequent remineralization. J Dent. 2018;75:34-40. doi: 10.1016/j.jdent.2018.05.002.

16. Marqués Martínez L, Leyda Menéndez AM, Ribelles Llop M, Segarra Ortells C, Aiuto R, Garcovich D. Dental erosion. Etiologic factors in a sample of Valencian children and adolescents. Cross-sectional study. Eur J Paediatr Dent. 2019;20(3):189-93. doi: 10.23804/ejpd.2019.20.03.04.

17. dos Santos O, Soares SD, Vieira E, Lisbôa L, Pinto D, da Costa Maciel A, et al. Consumo excessivo de bebidas potencialmente erosivas: implicações a saúde pública. Braz J Health Rev. 2019;2(4):2554-71 doi:10.34119/bjhrv2n4-027

18. Suh H, Rodríguez E. Determinación del ph y contenido total de azúcares de varias bebidas no alcohólicas: su relación con erosión y caries dental. Odontolnvestigación [Internet]. 2017 Mar [citado 24/02/2020];3(1):18-30 Disponible en: https://revistas. usfq.edu.ec/index.php/odontoinvestigacion/article/view/851

19. Reddy A, Norris DF, Momeni SS, Waldo B, Ruby JD. The pH of beverages in the United States. J Am Dent Assoc. 2016;147(4):255-63. doi: 10.1016/j. adaj.2015.10.019

20. Saads-Carvalho T, Lussi A. Chapter 9: Acidic beverages and foods associated with dental erosion and erosive tooth wear. Monogr Oral Sci. 2020;28:91-8. doi: 10.1159/000455376.

21. de Medeiros RA, Fernandes Neto, $J$ de $A$, de Vasconcelos Catão MHC. Evaluation of acid $\mathrm{pH}$ and erosive potential of alcoholic beverages. Arch Health Invest. 2018;7(7):254-7.

22. Barac R, Gasic J, Trutic N, Sunaric S, Popovic J, Djekic P, et al. Erosive effect of different soft drinks on enamel surface in vitro: application of stylus profilometry. Med Princ Pract. 2015;24(5):451-7. doi: 10.1159/000433435.

23. Iles $F$, Gutiérrez $O$. Sustancias químicas en bebidas gaseosas consumidas en Colombia y su relación con efectos sobre la salud. Rev Salud Hist Sanid on-line. 2016;11:51-66.

24. de-Medeiros RA, Fernandes-Neto J, Catão MH. Evaluation of acid pH and erosive potential of alcoholic beverages. Arch Health Invest. 2018;7:254-7 doi:10.21270/archi.v7i7.3117.

25. Gravelle BL, Hagen TW II, Mayhew SL, Crumpton B, Sanders T, Horne V. Soft drinks and in vitro dental erosion. Gen Dent. 2015;63(4):33-8 